\title{
RECENT TOPICS IN SPECTROSCOPY OF A-TYPE AND RELATED STARS
}

\author{
NLTE Analysis, Information-Extraction Technique, and Future Prospect
}

\author{
YOICHI TAKEDA \\ Institute of Astronomy, The University of Tokyo, \\ 2-21-1 Osawa, Mitaka, Tokyo, 181, Japan
}

\section{NLTE Abundance Determination}

The NLTE abundance analyses concerning late-B through A stars carried out before 1990 have been well summarized by Takada-Hidai (1991), which may be consulted for details; about 10 elements had been studied in NLTE by that time.

The notable NLTE studies concerning A-stars after 1990 are those of the Kiel group (cf. Stürenburg 1993, and the references therein), in connection with their extensive investigations on the $\lambda$ Boo and related weak-line stars. And one of their remarkable discoveries was the existence of C-Si anticorrelation in superficially normal A stars which has made an important impact on interpreting the origin of the abundance characteristics of weak-line stars; i.e., refractory elements such as $\mathrm{Fe}$ or $\mathrm{Si}$ have condensed into dust grains (considerable underabundance) while volatile elements like $\mathrm{C}, \mathrm{N}$, and $\mathrm{O}$ still remain in the gas phase (nearly normal abundance).

The Japanese group has also made some contribution to this field, in which their interest was focused mainly on the NLTE effect of neutral light-element lines such as C, N, O, and S in connection with analyses of near-IR spectra of CP stars (cf. Takada-Hidai and Takeda 1996, and the references therein). Especially, the near-IR triplet at $7773 \AA$ was found to be quite useful not only for oxygen abundance determination, but also for diagnosing the stellar parameters such as rotational velocity or turbulence (e.g., Takeda and Sadakane 1997).

These calculations, which exploited realistically large atomic models thanks to the recent progress in the numerical technique of radiative transfer along with the efficient computing facility nowadays, appear to have successfully clarified the importance of the NLTE effect. Though whether a line is affected by NLTE differs case by case, the wavelength region appears to be one of the important factors along with the line-strength. While LTE is generally a good approximation for deep-forming blue-region lines, near-IR lines (especially saturated ones) tend to suffer a considerable NLTE effect. And after applying the evaluated NLTE corrections, the abundances derived from different lines turn out to be consistent with each other. We may state, therefore, that recent NLTE calculations have arrived at a more or less quantitatively satisfactory level, compared to previous ones.

Yet, there still remain problems that must be overcome in order to make results more realistic and reliable. Above all important is the radiation field in UV, especially the region longward of the Lyman limit. A good example is the case of nitrogen (cf. Lemke and Venn 1996), where photoionization edges of important lower levels fall on the region of Lyman lines and edges of neutral carbon. This means that the abundance of carbon, along with the correct treatment of overlapping Lyman lines, is a key factor of significance for correctly simulating the UV radiation (and also the NLTE effect). We must here remind of the fact that the abundances of light elements (such as CNO) in chemically peculiar stars do not conform to those of heavier elements. Therefore, simple application of scaled-abundance models (such as previous ATLAS models) corresponding to the metallicity (often represented by the Fe abundance) to such $\mathrm{CP}$ stars may as well be rather questionable. However, now that opacity sampling models are now becoming available (cf. Kurucz 1996), by which models of arbitrary abundances can be constructed, we may hope that this problem will not be an essential obstacle any more. 


\section{Automatic Fitting of Synthetic Spectrum}

The spectral-synthesis technique has so far been widely used in an analysis of complexly merged spectra, where measuring equivalent widths of individual lines is hardly practicable. However, as those who have actually experienced it can easily realize, exact determination of various parameters (such as elemental abundances or the microturbulence velocity) by finding the best-fit by way of human eye is generally difficult and depends on individual's subjective judgement, especially when several parameters are intricately involved in the same feature.

However, a new way of spectral-synthesis analysis has recently become in practical use in the field of stellar spectroscopy. That is, an automatic finding of the best-fit theoretical spectrum with the observed one. Its basic principle is quite straightforward, which is essentially the numerical search for the parameter-solutions that minimize the $\chi^{2}$-residuals; and one can obtain subjectivefree solutions in a quick and accurate way. As a matter of fact, several codes devised for this purpose, each of which have their own unique characteristcs, appear to be successful in application to actual problems (e.g., Hill and Landstreet 1993; Takeda 1995; Cowley 1996; Valenti and Piskunov 1996; Hill et al. 1996).

Moreover, various kinds of flexible formulations are possible with this technique, depending on which parameters are regarded as variables to be established, thus providing prospect of wide applicability. We show here an example of such a merit: Let us recall that normalizing a spectrum with respect to the local continuum has been a common practice, occasionally being the cause of uncertainty. This was actually inevitable in the old days when photographic plates were the major observational device, because establishing the zero-intensity level was difficult. Yet, owing to the recent availability of linear detectors along with this kind of numerical approach, we can formulate the problem such that detector counts are directly compared with the model-fluxes by introducing a conversion multiplier as another variable (this is equivalent to regarding the continuum level as an unknown parameter to be determined). In this case, continuum placement prior to analysis may be circumvented (cf. Takeda 1995).

Now, the most common application of this automatic fitting technique is determinations of elemental abundances from a blended spectrum feature (while simultaneoully establishing the microturbulence), which is applicable also to broad-line stars of large $v_{\mathrm{e}} \sin i$. Yet should it be stressed that above all important is to use an accurate set of $g f$ values in this case.

This can also be effectively applied to the detailed profile-analysis, which aims at detecting the delicate second-order effect, such as separating the rotational velocity from the turbulent broadening, estimating the inclination of rotation-axis, or studying the nature of differential rotation.

Another useful possibility of application would be the determination of astrophysical (empirical) $g f$ values from a severely merged spectrum, which can be accomplished by regarding each $\epsilon g f$ values of appreciable lines as variables to be established.

As has been listed above, this automated fitting method can be a promising tool. However, it is by no means a fool-proof black box. In order for its successfull application, we have to set up the problem very carefully, so that the existence of best-fit solution may be guaranteed. Important points that should be kept in mind when applying this technique are such as these:

- Spectral-line database is complete and accurate?

- Parametrization is adequate?

- Modeling of the theoretical spectra realistic enough?

\section{Stellar Spectroscopy Toward the Future}

One of the most important features in the astronomy of 21 th centry is the $10 \mathrm{~m}$-class big tclescopes of large light-collectivity. coupled with efficient solid-state detectors of wide wavelength coverage as well as of large format. And in the field of high-dispersion stellar spectroscopy, we can then expect, realization of:

- Increasing number of observable objects

- Wide spectral range available 
- Very high S/N ratio

- Multi-object spectroscopy (e.g., fiber-feed spectrograph).

This naturally indicates the necessity of efficient and accurate extraction of huge amount of information, which should be earnestly considered. ¿From this point of view, it may be expected that spectral-synthesis equipped with automatic fitting, which has been described in section 2, can serve as a useful and promising way of data-processing in spectroscopic analysis.

Then, what about the prospect of the theoretical side? Owing to the recent progress of computing facility and of the technique of numerical radiative transfer, we have sufficient tools for carring out more sophisticated calculations than have ever been tried, such as NLTE line-formation calculation in inhomogeneous atmosphere or in the presence of a magnetic field. It seems, however, that strong motivation from observational side has been lacking. Namely, as far as analysis of moderate-quality spectrum is concerned, invoking the classical treatment suffices in most cases without revealing serious problems, and one tends to settle in the world of good old days.

Nevertheless, the situation appears to be gradually changing thanks to the advent of recent ultra-high S/N spectroscopy. A good example is the flat-bottomed profile of very weak lines found in the very high $\mathrm{S} / \mathrm{N}$ (several thousands) spectrum of Vega, which is diffficult to understand in the framework of the classical modeling. And it was Gulliver et al. (1994) who showed that this anomolous shape can be explained by the pole-on model of a rapidly rotating star (i.e., latitudedependent physical condition). This is an instructive fact, which clearly shows that we can not avoid the problem of inhomogeneity (i.e., non-classical modeling), if we step into the world of ultra-high signal-to-noise ratio.

We may possibly see light in this direction for the future stellar-spectroscopy. Namely, what is needed for a breakthrough in this field is such observational data

- showing crucial breakdown of the conventional modeling

- containing wealthy information that can be compared to sophisticated theory

which attractively inspire theoretician's volution and/or ambition.

Hence, we may expect that more and more analyses of very-high quality spectra would lead to important progress in stellar spectroscopy. At present, such observation is realized only for firstmagnitude class stars such as Vega. But with the help of 10m-class telescopes, it is certain that the candidates of such ultra-high quality observation can be considerably extended. Admittedly, the general trend of modern astronomy is "toward fainter objects", but we should also keep in mind the importance of "toward fainter features". In fact, we would be greatly benefited if large telescopes could also be exploited for this kind of, say, microscopic studies rather than telescopic.

\section{References}

Cowley C.R. 1996, in Model Atmospheres and Spectrum Synthesis, ASP Conference Series Vol.108, eds S.J. Adelman, F. Kupka, and W.W. Weiss, p. 170

Gulliver A.F., Hill G., Adelman S.J. 1994, Astrophys. J. 429, L81

Hill G., Gulliver A.F., Adelman S.J. 1996, in Model Atmospheres and Spectrum Synthesis, ASP Conference Series Vol.108, eds S.J. Adelman, F. Kupka, and W.W. Weiss, eds S.J. Adelman, F. Kupka, and W.W. Weiss, p.184 Hill G.M., Landstreet J.D. 1993, Astron. Astrophys. 276, 142

Kurucz R.L. 1996, in Model Atmospheres and Spectrum Synthesis, ASP Conference Series Vol.108, eds S.J. Adelman, F. Kupka, and W.W. Weiss, eds S.J. Adelman, F. Kupka, and W.W. Weiss, p.160

Lemke M., Venn K. 1996, Astron. Astrophys. 309, 558

Stürenburg S. 1993, Astron. Astrophys. 277, 139

Takada-Hidai M. 1991, in Evolution of Stars: The Photospheric Abundance Connection, Proc. IAU Symp.145, eds. G. Michaud and A. Tutukov (Kluwer, Dortrecht), p.137

Takada-Hidai M., Takeda Y. 1996, Publ. Astron. Soc. Japan, 48, 739

Takeda Y. 1995, Publ. Astron. Soc. Japan, 47, 287

Takeda Y., Sadakane K. 1997, Publ. Astron. Soc. Jopan, 49, 367

Valenti J.A., Piskunov N. 1996, Astron. Astrophys. Suppl. 118, 595 